\title{
Epipolar Plane Diffusion: An Efficient Approach for Light Field Editing
}

\author{
Oriel Frigo \\ oriel.frigo@inria.fr \\ Christine Guillemot \\ christine.guillemot@inria.fr
}

Inria

Centre Rennes

Bretagne, France

\begin{abstract}
This paper presents a novel approach for light field editing. The problem of propagating an edit from a single view to the remaining light field is solved by a structure tensor driven diffusion on the epipolar plane images. The proposed method is shown to be useful for two applications: light field inpainting and recolorization. While the light field recolorization is obtained with a straightforward diffusion, the inpainting application is particularly challenging, as the structure tensors accounting for disparities are unknown under the occluding mask. We address this issue with a disparity inpainting by means of an interpolation constrained by superpixel boundaries. Results on synthetic and real light field images demonstrate the effectiveness of the proposed method.
\end{abstract}

\section{Introduction}

With the increasing popularity of computational photography brought by light field [ष] cameras such as Lytro [ $[\mathbf{⿴ 囗 十}]$ and Raytrix, simple and intuitive editing of light field images is becoming a feature of high interest for general users. Light field editing can be combined with the traditional refocusing feature, allowing a user to include or remove objects from the scene or change the color, contrast or other features of the scene objects.

A simple approach for editing a light field image can be obtained with an edit propagation, where first a particular subaperture view is edited (most likely the center one) and then a coherent propagation of this edit is performed through the other views [ $\square]$ [ $\square]$ [ $[\mathbb{Q}]$. This problem is particularly challenging for the task of inpainting, as the disparity field is unknown under the occludding mask.

This paper provides a novel and efficient solution to this problem, where we address an angularly coherent propagation of local edits through an approach we call epipolar plane diffusion, as it performs an anisotropic diffusion on epipolar plane images. Anisotropic diffusion has been extensively applied to solve image editing problems such as inpainting, due to properly modeling piecewise smooth image content. However, diffusion approaches met a diminished usage in inpainting due to their limited capability of reconstructing image textures.

In this work, we revisit anisotropic diffusion, showing that tensor driven diffusion is an appropriate model to be applied to Epipolar Plane Images (EPI) derived from light fields. 
Since EPIs are piecewise smooth and have no complex texture content, tensor driven diffusion is naturally suited for inpainting the EPIs as an efficient technique to obtain a coherent edit propagation.

To summarize, the present work makes the following contributions, which are novel for the best of our knowledge:

- An application of tensor-based diffusion to inpaint epipolar plane images and obtain angularly coherent edit propagation;

- A new method to obtain disparity inpainting, based on a superpixel-guided interpolation of structure tensors.

The paper is organized as follows: Section 2 discusses previous work related to light field editing, inpainting and anisotropic diffusion; Sec. 3 presents our epipolar plane diffusion method; Sec. 4 discusses the application of our method to light field recolorization and inpainting; Sec. 5 demonstrates the efficiency of the proposed algorithm through experiments with synthetic and real light field images. Finally, Sec. 6 concludes the paper with final considerations and suggestions of future work.

\section{Related Work}

\subsection{Light field editing}

Light field edit propagation has been first discussed by [ $\mathbb{} \mathbf{Q}]$, where the authors describe an approach using a 3D voxel-based model of the scene with an associated radiance function to propagate pixel edits and illumination changes in a consistent manner from one view to the other views of the light field. The authors in [ $\square]$ ] extend the 2D image stroke-based edit propagation method of $[\square]$ to light fields. To overcome the computational burden inherent to light field data, the edits are propagated in a downsampled version of the light field. In [ $\mathrm{\theta}$, a method based on a reparametization of the light field is proposed to better preserve the coherence of the edits in the angular domain. These papers differ from our work as they deal with stroke-based editing and were not intended for more complex editing tasks such as inpainting.

The recent article of [四] share some similarities with our method as the central view is edited beforehand, using a 2D patch-based method. The offsets between the filled patches and their best match in the known region are propagated to other views, with the help of disparity information, in order to fill them in a consistent manner. This work is applied to light field recolorization and inpainting, however the specific problem of disparity map inpainting is not handled.

\subsection{Light field Inpainting}

The term inpainting was first used by Bertalmio et. al in [ [ $]$ ] and is borrowed from its use in fine arts for the process of restoring damaged parts of images. The inpainting problem was formulated as recovering a selected region $\Omega_{m}$ (occluding mask) by propagating the intensities from the boundary $\partial \Omega_{m}$ to the center of the unknown area along similar level lines (called isophotes). A number of works [四] [0] [四] considered this propagation as an anisotropic diffusion which is the solution of a partial differential equation (PDE). These 
inpainting methods based on PDEs are able to reconstruct geometry, but not texture. Nevertheless, we show in this work that they are adequate for the inpainting of epipolar plane images, which have no texture content.

A second wave of inpainting methods appeared after the work of Criminisi et. al [四], which was influenced by patch-based texture synthesis [ㅁ] [ㅁ] . Patch-based approaches are able to inpaint textures but can fail to correctly reconstruct geometry, which motivated some works to combine patch-based and PDE-based inpainting [日].

With the advances in machine learning, in particular convolutional neural networks and auto-encoders, inpainting methods such as [四] can correctly reconstruct image geometries, textures and semantic content. Such methods could be combined with the approach introduced in this paper, as we propose to propagate a $2 \mathrm{D}$ inpainting computed on the central view (for instance obtained with [四]) to the complete set of light field views. An extensive survey on 2D inpainting can be found in [प⿴囗⿰丿㇄口].

With respect to light field inpainting, there are far fewer works in the literature. In [ $\mathrm{Z}]$, the authors extend the exemplar-based method of [ $⿴ 囗 ⿱ 一 一])$ for light field inpainting. One of the views is inpainted first using a 2D method. For the other views, instead of searching a best matching patch in the known region of the view to inpaint, the patch is searched in the first inpainted view in order to ensure a better consistency across views. A 4D patch-based method is also considered in [ $\mathrm{B}]$, where consistency is ensured by minimizing a 4D patch bi-directional similarity measure. In the variational framework proposed by [ㅁ] , various inverse problems are solved, including inpainting. By exploiting depth information, they define constraints on the structure of the epipolar plane images of the light field, thus ensuring a consistent inpainting accross views. Inpainting of the disparity map is briefly discussed by the authors, where a linear interpolation is followed by variational regularization. We note in our work that an unconstrained interpolation of the disparity map such as performed by [ $\square$ ] can lead to depth inconsistency if the inpainting mask spread across different depth layers. To handle this problem, we propose a tensor interpolation constrained by a segmentation of the edited central view.

\section{Epipolar Plane Diffusion}

In a nutshell, our approach imposes angular coherency for light field editing by inpainting the epipolar plane images. Inpainting is modeled as an anisotropic diffusion on EPIs, guided by structure tensors.

Let $L: \Omega \times \Pi \rightarrow \mathbb{R}^{3}$ be a light field with spatial coordinates $(x, y) \in \Omega$, spatial dimensionality $|\Omega|=M \times N$, angular coordinates $(s, t) \in \Pi$ and angular dimensionality $|\Pi|=P \times Q$. In other words, the light field image $L$ can be seen as a matrix of $P \times Q$ views.

The central view of $L$ is denoted $I_{c}:=L\left(x, y,\left\lceil\frac{P}{2}\right\rceil,\left\lceil\frac{Q}{2}\right\rceil\right)$. In this paper, it is assumed that the central view $I_{c}$ has been edited beforehand and the goal is to obtain angular coherence between the edited central view $\hat{I}_{c}$ and the remaining views.

An EPI is a spatio-angular slice from the light field, obtained by fixing one of the spatial coordinates and one of the angular coordinates. Assuming we fix $y:=y *$ and $t:=t *$, an EPI is an image defined as $E_{y * t *}:=L(x, y *, s, t *)$. It is well known that the slopes of isophote lines in an EPI give the amount of disparity between the views belonging to the same light field row or column. Since the structure tensors computed from an EPI contain the gradient magnitudes and orientations, they naturally encapsulate inter-view disparities. Our goal here is to diffuse the edited part from the central view to the peripheric views, ensuring that this 


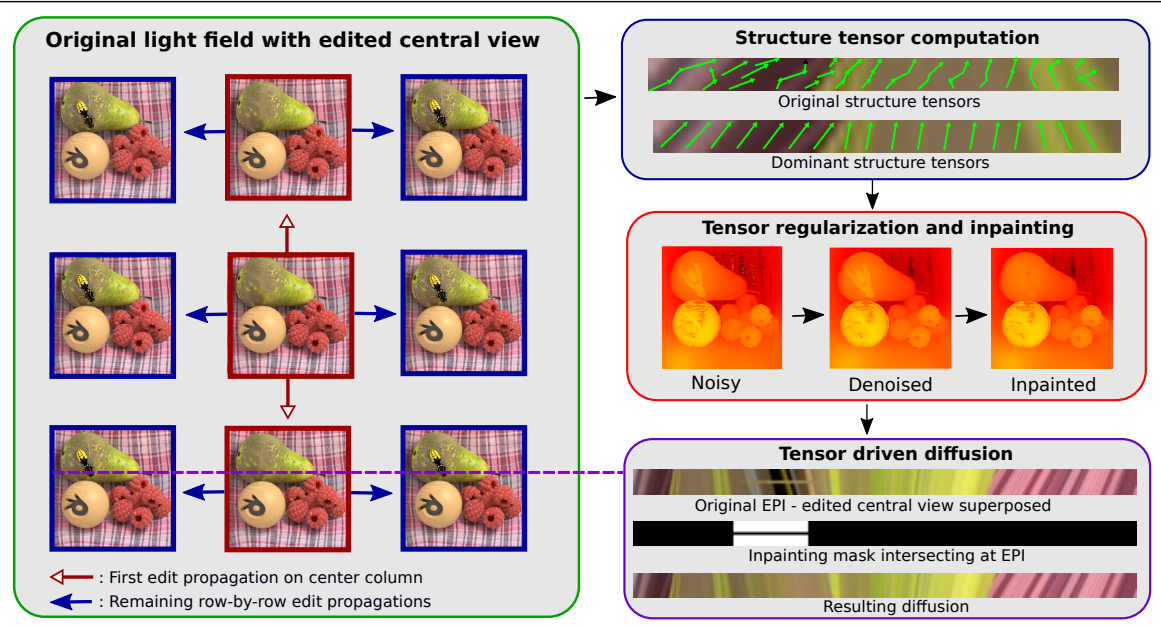

Figure 1: Overview of the proposed method. In the green box, it is illustrated a light field with an inpainted central view. We illustrate the original light field after a first edit propagation, which is performed through the center column of views (red arrows). Remaining edit propagations are performed row-by-row (blue arrows). The remaining boxes illustrate the steps of epipolar plane diffusion: Structure tensor computation (Eq. 1) where we obtain dominant structure tensors (Eq. 2); From dominant structure tensors we compute a spatial regularization (Eq. 3) and tensor inpainting (Eq. 6); and finally a tensor driven diffusion (Eq. 4) on EPIs. The output views are reconstructed from the resulting diffusions on the EPIs.

diffusion follows the orientation of the EPI structure tensors.

An overview of our method is illustrated in Fig. 1. Note that the EPIs in a light field connect together the subaperture views from the same row (or column) of the light field, accounting for only horizontal (or only vertical) disparities. Our proposed epipolar plane diffusion allows to propagate an editing from the center view of a light field row (or column), to the other views of same row (or column). There are different possibilities for the propagation order, here we arbitrarily first compute a propagation through the central column of the light field, and in the sequence we compute a row-by-row tensor driven diffusion.

\subsection{Tensor Driven Diffusion}

In this work, we rely on the smoothed structure tensors computed from the EPI to estimate the diffusion tensors which are related to the disparity between views. For an EPI $E_{y *, t *}$ and Gaussian kernel $g_{\sigma}$ with variance $\sigma^{2}$, the smoothed structure tensor at coordinate $(x, s)$ is given by:

$$
T_{\sigma}(x, s)=\nabla E_{y *, t *}(x, s) \nabla E_{y *, t *}(x, s)^{T} * g_{\sigma} .
$$

To ensure that the tensor driven diffusion is performed along a single coherent direction per column of the EPI, we compute one dominant structure tensor $\bar{T}_{\sigma}(x)$ for each spatial coordinate $x$ as a weighted average:

$$
\bar{T}_{\sigma}(x)=\frac{\sum_{s=1}^{P} A(x, s) T_{\sigma}(x, s)}{\sum_{s=1}^{P} A(x, s)},
$$

where $A(x, s)=\left(\frac{\lambda_{+}-\lambda_{-}}{\lambda_{+}+\lambda_{-}}\right)^{2} \in[0,1]$ measures the anisotropy of the structure tensor at coordinate $(x, s)$ and $\lambda_{+}, \lambda_{-}$are respectively the largest and the smallest eigenvalues computed 
from $T_{\sigma}(x, s)$. Basically, structure tensors that are anisotropic indicate higher coherence, thus we enforce them to receive larger weights in the estimation of a dominant structure tensor.

The orthonormal eigenvectors $\theta_{+} \in \mathbb{R}^{2}$ and $\theta_{-} \in \mathbb{R}^{2}$ computed from structure tensors are used in a number of works to drive an anisotropic diffusion. Here we are mostly interested in $\theta_{-}$, which corresponds to the smallest eigenvalue $\lambda_{-}$. Note that $\theta_{-}$is the eigenvector that gives the direction of constant intensity lines, and as such it can be considered as the isophote eigenvector, being directly related to the disparities between views.

As noted by $[\mathbb{Q}]$, the pointwise structure tensors computed from an EPI may be too noisy for a reliable disparity estimation. The gaussian smoothing and the weighted average reduces the noise, but a further regularization is necessary. From the observation that disparity maps are approximately piecewise constant, we compute a spatial regularization by performing a Total Variation (TV-L1) denoising [ $[\square]$ on the eigenvector field $\Theta_{-}: \Omega \rightarrow \mathbb{R}^{2}$, which is the set of all isophote eigenvectors computed from the dominant structure tensors $\bar{T}_{\sigma}$. Given a noisy observed $\Theta_{-}$, we search for

$$
\hat{\Theta}_{-}=\underset{\hat{\Theta}_{-}}{\arg \min }\left\|\nabla \hat{\Theta}_{-}\right\|+\lambda_{t v}\left\|\hat{\Theta}_{-}-\Theta_{-}\right\|
$$

where $\lambda_{t v}$ is the regularization parameter, $\lambda_{t v}:=0.5$ in our experiments. In practice, minimization is obtained by the Primal-Dual algorithm [日].

We note that structure tensors computed from EPIs were already proposed in the previous work of [ $\square]$ for light field disparity estimation in a variational framework. Our work differs from that as we use these structure tensors as diffusion tensors in an anisotropic diffusion. As mentioned in Section 2, in this paper we build upon the vector-valued regularization PDE of Tschumperlé and Deriche [ $\square$ ] to perform a tensor driven diffusion. The diffusion over the EPI $E_{y *, t *}$ evolves as follows:

$$
\frac{\partial E_{y *, t *}}{\partial t}=\operatorname{trace}\left(T H_{t}\right)
$$

where $T=f\left(\bar{T}_{\sigma}\right)$ is the tensor field driving the diffusion and $H_{t}$ denotes the hessian of $E_{y *, t *}$. Note that different propositions for $f$ exist in the literature, depending on the reconstruction problem to be solved. Here we are interested in a setting where diffusion should occur only in the direction of the isophote eigenvector, where $T$ is a diffusion tensor reconstructed as $T=\hat{\Theta}_{-} \hat{\Theta}_{-}^{T}$.

The tensor based diffusion is performed on the region inside the inpainting mask and is implemented by iterative finite differences. In Figure 1 (purple box) we illustrate the diffusion on the EPI, where an EPI mask can be seen. Differently to typical inpainting applications where image intensities are diffused from the border to the center of $\Omega_{m}$, here we have the inverse setting, where the center of the mask is initially known (the central view intensities) and we desire to propagate these intensities into the periphery of the mask. In order to have known intensities on the EPI mask boundaries, we first shift the intensities from the central row to the first and the last rows of the EPI mask. This shift is performed based on the disparity measure $\Delta=\frac{\hat{\theta}_{-}^{x}}{\hat{\theta}_{-}^{s}}$ which accounts to the ratio between the $x$ and $s$ components of the isophote eigenvector $\hat{\theta}_{-} \in \hat{\Theta}_{-}$. Then, the remaining rows of the EPI are completed by diffusion, which prevents from line aliasing. 


\section{Applications}

In this section, we discuss how our method can be used for light field editing applications, in particular recolorization and inpainting. Both applications are based on the idea of first editing the central light field view, either manually or using available algorithms and then propagating this edit to the remaining light field views. We consider a local editing, where a specific region of the central light field view is edited, this region is given by a mask $\Omega_{m} \subset \Omega$.

\subsection{Light Field Recolorization}

Recolorization consists in modifying the color palette of an image, either manually or based on color transfer algorithms which rely on example images. Here we are interested in local recolorization, where color editing occurs inside a mask $\Omega_{m}$.

Local recolorization is obtained in a straightforward manner with our proposed epipolar plane diffusion, based on the procedures described in Section 3. Results can be seen in Section 5.

\subsection{Light Field Inpainting}

The light field inpainting application is particularly challenging, as the disparities between the views are not determined for the pixel coordinates which are lying on the inpainting mask $\Omega_{m}$. It follows that the disparity map should be inpainted for a correct propagation of the inpainted region from the central view to the peripheric views.

By means of disparity interpolation, we can enforce the disparities inside the inpainting mask to be similar to the disparities surrounding the mask. However, if we naively interpolate the unknown disparities based on the nearest known disparities, depth boundaries may be violated, and disparity values from different depth layers may be mixed together in the interpolation.

To overcome this issue, we assume that inside the inpainting mask, disparity clusters are likely to have shape and boundaries similar to the known color clusters. In practice, we consider a segmentation of the edited central view $\hat{I}_{C}$ into SLIC superpixels [四], where each superpixel account for a color cluster on $\hat{I}_{c}$. Superpixels are an adequate image partitioning for this problem since their boundaries tend to respect object boundaries in the image. Hence, the main purpose of superpixel segmentation is to enforce the disparity interpolation to respect depth boundaries in the light field.

We consider a superpixel set $S=\left\{s_{i}\right\}_{i=1}^{N_{s}}$ which is a spatial partition of the inpainted central view $\hat{I}_{c}$. Then, the unknown disparity at a pixel is obtained by a linear combination of the nearest known disparities which belong to the same superpixel $s_{i}$.

A preliminary superpixel merging is required to ensure that for every superpixel, at least $K$ pixels ( $K:=4$ in our experiments) have known disparities from which the interpolation can be computed. Formally, if we assume that a superpixel $s_{i} \in S$ has less than $K$ known disparities, then $s_{i}$ is merged to the most similar neighbor superpixel $s_{j} \in \mathcal{N}_{s_{i}}$, where $\mathcal{N}_{s_{i}}$ is the neighborhood of $s_{i}$. The most similar superpixel is given by

$$
\underset{s_{j} \in \mathcal{N}_{s_{i}}}{\arg \min }\left\|\mu_{s}\left(s_{i}\right)-\mu_{s}\left(s_{j}\right)\right\|+\left\|\sigma_{s}^{2}\left(s_{i}\right)-\sigma_{s}^{2}\left(s_{j}\right)\right\|
$$

where $\mu_{s}($.$) is the mean superpixel color and \sigma_{s}^{2}($.$) is the superpixel variance, which are$ both computed in CIELAB color space. Finally, the interpolated eigenvector at a masked 


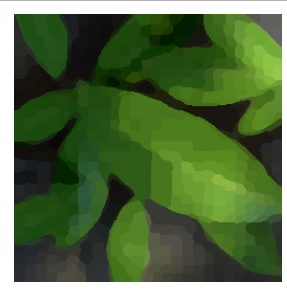

a)

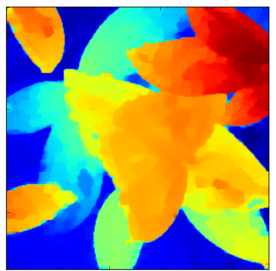

b)

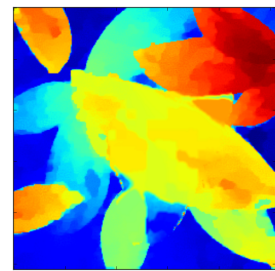

c)

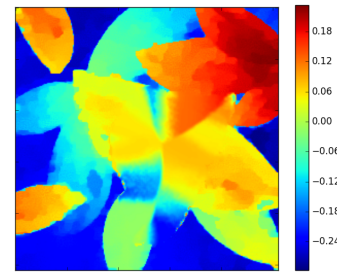

d)

Figure 2: Illustration of disparity inpainting. a) Superpixel segmentation of inpainted central view. b) Disparity map computed from original light field. c) Disparities computed with our superpixel guided interpolation. d) Disparities computed with a non-constrained interpolation.

coordinate $\mathbf{p}=\left(p_{x}, p_{y}\right) \in s_{i} \cap \Omega_{m}$ is given by

$$
\hat{\Theta}_{-}(\mathbf{p})=\sum_{\mathbf{q} \in \mathrm{NN}(\mathbf{p})} w(\mathbf{p}, \mathbf{q}) \hat{\Theta}_{-}(\mathbf{q}),
$$

where $\mathrm{NN}(\mathbf{p})$ is the set of $K$ nearest neighbors of $\mathbf{p}$ and $w$ is an inverse-distance weighting factor. The weights are given by

$$
w(\mathbf{p}, \mathbf{q})=\frac{1}{Z} \exp \left(-\frac{d(\mathbf{p}, \mathbf{q})}{\sigma_{\mathrm{NN}}^{2}}\right)
$$

where $Z$ is a normalization factor that sums up weights to 1 and $\sigma_{\mathrm{NN}}^{2}$ is the variance of the nearest neighbor distances. The set of nearest neighbors of $\mathbf{p}$ is given by

$$
\mathrm{NN}(\mathbf{p})=\underset{\mathbf{q}_{k} \in \tilde{s}_{i}}{\arg \min } d\left(\mathbf{p}, \mathbf{q}_{k}\right) \quad k=\{1, \ldots, K\}
$$

where $\tilde{s}_{i}=s_{i} \backslash s_{i} \cap \Omega_{m}$ is the set of non-masked coordinates at superpixel $s_{i}$, and $d$ is a distance that takes into account spatial and color differences:

$$
d(\mathbf{p}, \mathbf{q})=(\mathbf{p}-\mathbf{q})^{2}+\left(\hat{I}_{c}(\mathbf{p})-\hat{I}_{c}(\mathbf{q})\right)^{2} .
$$

In Figure 2 we illustrate the super-pixel based eigenvector interpolation. It can be noted that our superpixel-guided interpolation correctly preserves most shapes in the disparity map, while an interpolation without any constraint tends to violate depth boundaries.

\section{Results and Discussion}

This section presents experiments with the method proposed in this paper. In Fig. 3, we show the results of our approach applied to recolorization and inpainting of the synthetic light field "Butterfly". For this experience, first the central view is recolored and inpainted manually with an image manipulation tool, and then the editing is propagated through the light field with our method. Note that the inpainting propagation is challenging for this light field, since the inpainting mask crosses different depth layers (leaves and ground). Still, our method succesfully propagates the recolorization and inpainting through all the views, resulting in a coherent image refocusing. 

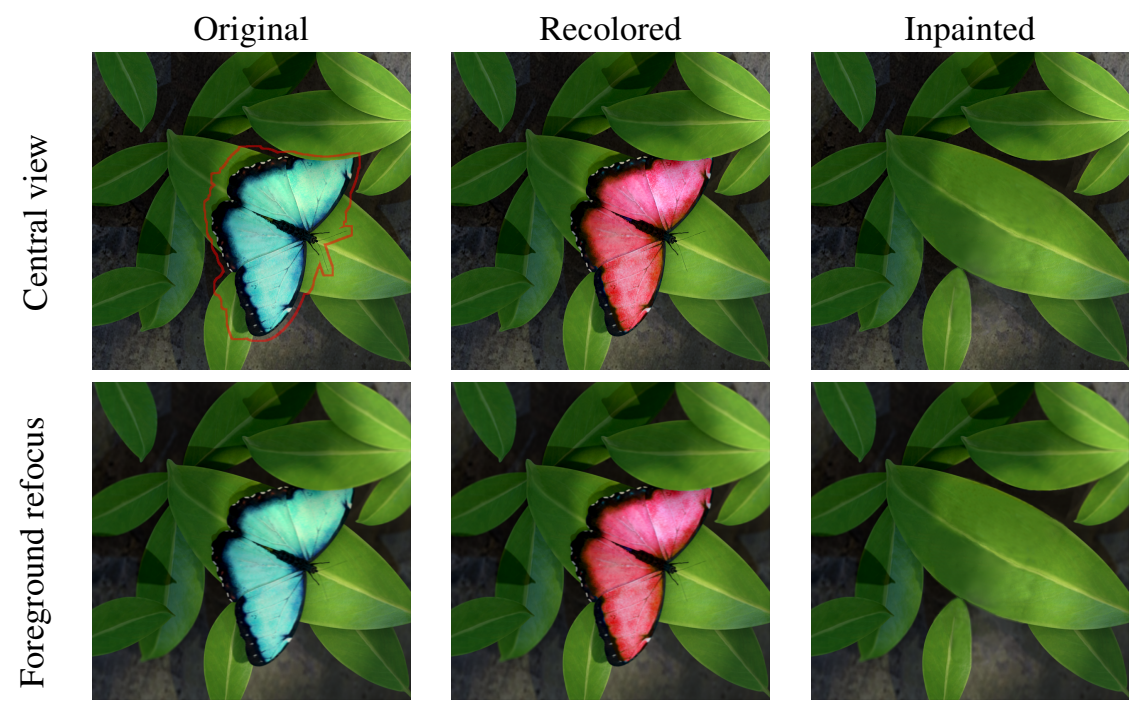

Figure 3: Results of edit propagation with our method on synthetic light field. The inpainting mask contour is represented in red color.

Experiments with two real light fields, obtained with the camera Lytro 1.0 and rendered with the pipeline of $[\square]$, are shown in Fig. 4. To alleviate color fluctuations between views, we first perform a histogram specification between the central and remaining views. The central view is inpainted by the patch-based method of $[\square]$. Since we do not have access to the implementation of the few previous works on light field edit propagation, we compare our method with a baseline algorithm, which performs edit propagation by view warping based on optical flow. We estimate the optical flows between the views with the state-of-the-art method of $[\square]$ and warp the edited central view based on the estimated disparity fields.

In Fig. 4, it can be seen that the edit propagation obtained with the baseline fails to correctly propagate the edit on the central view, resulting in a refocusing with noticeable inconsistency. Note that the inpainting mask is still visible after refocusing, where foreground and background areincoherently mixed. On the other hand, our method allows for a coherent edit propagation with correct refocusing at foreground and background.

We note that our unoptimized implementation in python takes in average one minute to propagate an inpainting to all light field views (Lytro 1.0 image), but our algorithm could greatly benefit from parallel processing. We invite the reader to access our project website (http://oriel.github.io/epipolar_plane_diffusion.html), where we provide more results obtained with our method.

\section{Conclusion}

This paper presented a new approach for the propagation of light field edits based on diffusion on epipolar plane images. Our results on synthetic and real light field images have demonstrated the efficiency of the proposed method. This work has been focused on local light field editing, where a portion of the central view is edited, as shown in the inpainting and recolorization applications. Future work may extend the approach for global light field 

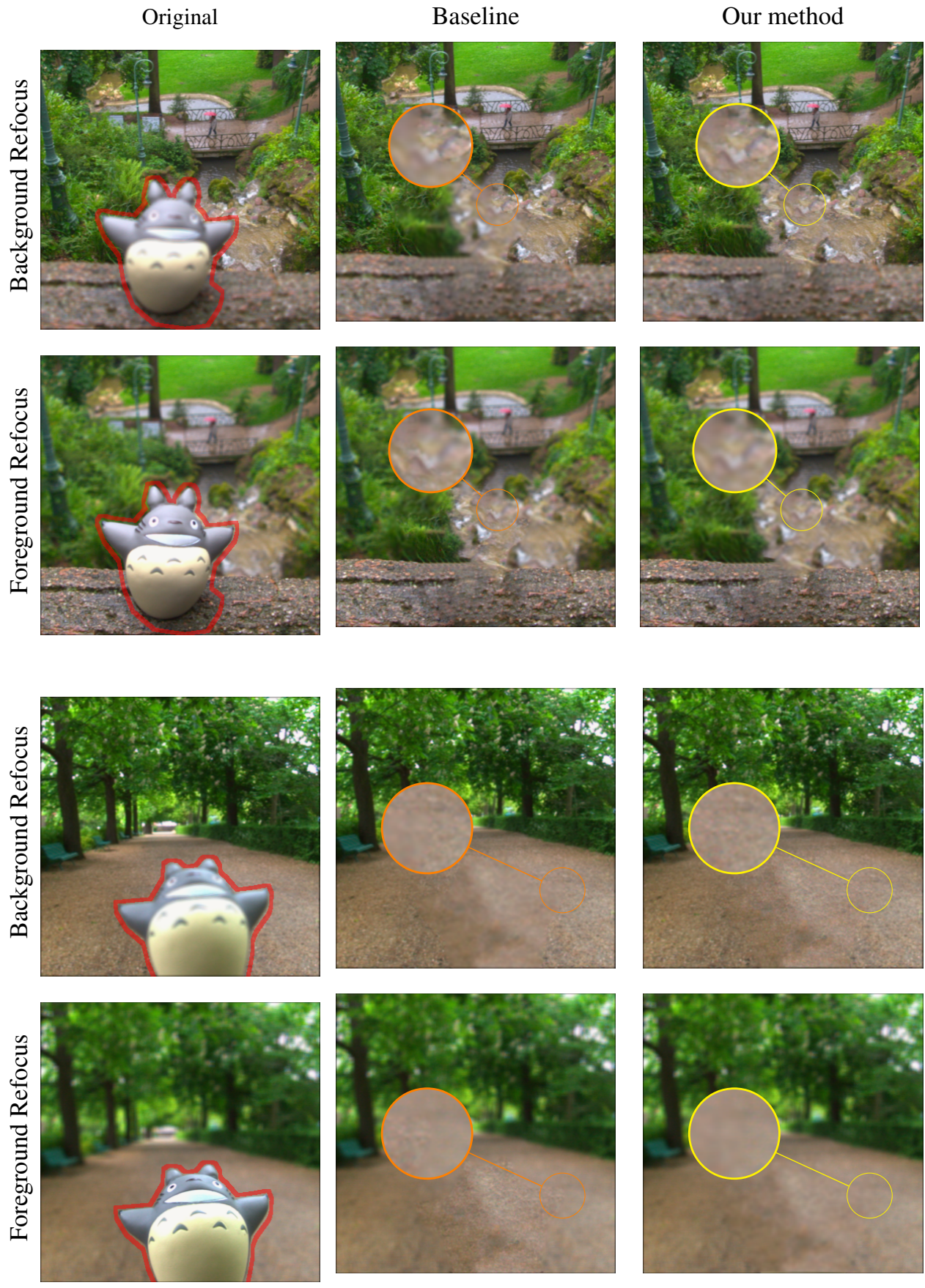

Figure 4: Inpainting on real light fields, performed with a baseline and our method. It can be particularly noted in the zoomed areas that the refocusing using the baseline method (orange circles) incorrectly mixes "in focus" and "out of focus" pixels, while the light field inpainted with our method (note the yellow zoom circles) results in consistent refocusing. The inpainting mask contour is represented in red color. 
processing, for which our approach could be used to guarantee angular coherence for other tasks such as segmentation and super-resolution. For that, occlusions should be carefully handled in the epipolar plane diffusion.

Finally, we believe this paper may inspire new approaches for using tensor diffusion with light field images. For instance, 4D structure tensors could be employed to obtain simutaneously spatial and angular coherence in light field reconstruction problems.

\section{Acknowledgements}

This work was supported by the EU H2020 Research and Innovation Programme under grant agreement No 694122 (ERC advanced grant CLIM).

\section{References}

[1] Radhakrishna Achanta, Appu Shaji, Kevin Smith, Aurélien Lucchi, Pascal Fua, and Sabine Süsstrunk. Slic superpixels compared to state-of-the-art superpixel methods. IEEE Trans. Pattern Anal. Mach. Intell., 34(11):2274-2282, 2012. doi: 10.1109/ TPAMI.2012.120. URL http: / / dx. doi.org/10.1109/TPAMI . 2012.120.

[2] Xiaobo An and Fabio Pellacini. Appprop: all-pairs appearance-space edit propagation. ACM Trans. Graph., 27(3):40:1—-40:9, 2008. doi: 10.1145/1360612.1360639. URL http://doi.acm.org/10.1145/1360612.1360639.

[3] Hongbo Ao, Yongbing Zhang, Adrian Jarabo, Belen Masia, Yebin Liu, Diego Gutierrez, and Qionghai Dai. Light Field Editing Based on Reparameterization, pages 601-610. Springer International Publishing, Cham, 2015. ISBN 978-3-319-240756. doi: 10.1007/978-3-319-24075-6_58. URL http://dx.doi.org/10.1007/ $978-3-319-24075-6\{\ldots\} 8$.

[4] Marcelo Bertalmio, Guillermo Sapiro, Vincent Caselles, and Coloma Ballester. Image inpainting. Proceedings of the 27th annual conference on Computer graphics and interactive techniques SIGGRAPH 00, 2(5):417-424, 2000. ISSN 19410042. doi: 10.1145/344779.344972. URL http://portal.acm.org/citation.cfm? doid=344779.344972.

[5] Aurélie Bugeau and Marcelo Bertalmio. Combining Texture Synthesis and Diffusion for Image Inpainting. In VISAPP 2009 - Proceedings of the Fourth International Conference on Computer Vision Theory and Applications, pages 26-33, Portugal, 2009. URL https: / / hal.archives-ouvertes.fr/hal-00551587.

[6] Antonin Chambolle. An algorithm for total variation minimization and applications. $J$. Math. Imaging Vis., 20(1-2):89-97, January 2004. ISSN 0924-9907. doi: 10.1023/B: JMIV.0000011325.36760.1e. URL http://dx.doi.org/10.1023/B: JMIV . 0000011325.36760 .1 e.

[7] Tony F. Chan, Sung Ha Kang, Kang, and Jianhong Shen. Euler's elastica and curvature based inpaintings. SIAM J. Appl. Math, 63:564-592, 2002. 
[8] Gaurav Chaurasia, Sylvain Duchêne, Olga Sorkine-Hornung, and George Drettakis. Depth synthesis and local warps for plausible image-based navigation. ACM Trans. Graph., 32(3):30:1-30:12, 2013. doi: 10.1145/2487228.2487238. URL http:// doi.acm.org/10.1145/2487228.2487238.

[9] Ke-Wei Chen, Ming-Hsu Chang, and Yung-Yu Chuang. Light field image editing by 4d patch synthesis. In 2015 IEEE International Conference on Multimedia and Expo, ICME 2015, Turin, Italy, June 29 - July 3, 2015, pages 1-6. IEEE, 2015. ISBN 978-14799-7082-7. doi: http://dx.doi.org/10.1109/ICME.2015.7177447.

[10] Antonio Criminisi, Patrick Perez, and Kentaro Toyama. Region filling and object removal by exemplar-based image inpainting. IEEE Transactions on Image Processing, 13/9:1200-1212, September 2004. URL https://wWw.microsoft.com/en-us/research/publication/ region-filling-and-object-removal-by-exemplar-based-inpainti

[11] Maxime Daisy, David Tschumperlé, and Olivier Lézoray. A fast spatial patch blending algorithm for artefact reduction in pattern-based image inpainting. In SIGGRAPH Asia 2013 Technical Briefs, SA '13, pages 8:1-8:4, New York, NY, USA, 2013. ACM. ISBN 978-1-4503-2629-2. doi: 10.1145/2542355.2542365. URL http: //doi.acm.org/10.1145/2542355.2542365.

[12] Donald G. Dansereau, Oscar Pizarro, and Stefan B. Williams. Decoding, calibration and rectification for lenselet-based plenoptic cameras. In Proceedings of the 2013 IEEE Conference on Computer Vision and Pattern Recognition, CVPR '13, pages 10271034, Washington, DC, USA, 2013. IEEE Computer Society. ISBN 978-0-7695-49897. doi: 10.1109/CVPR.2013.137. URL http://dx. doi .org/10.1109/CVPR. 2013.137.

[13] Alexei A. Efros and William T. Freeman. Image quilting for texture synthesis and transfer. In Proceedings of the 28th Annual Conference on Computer Graphics and Interactive Techniques, SIGGRAPH '01, pages 341-346, New York, NY, USA, 2001. ACM. ISBN 1-58113-374-X. doi: 10.1145/383259.383296. URL http://doi . $\mathrm{acm}$. org/10.1145/383259.383296.

[14] Alexei A. Efros and Thomas K. Leung. Texture synthesis by non-parametric sampling. In Proceedings of the International Conference on Computer Vision-Volume 2 Volume 2, ICCV '99, pages 1033-, Washington, DC, USA, 1999. IEEE Computer Society. ISBN 0-7695-0164-8. URL http://dl . acm.org/citation. cfm?id= 850924.851569 .

[15] Bastian Goldluecke and Sven Wanner. The variational structure of disparity and regularization of $4 \mathrm{~d}$ light fields. In IEEE Conference on Computer Vision and Pattern Recognition, Portland, OR, USA, June 23-28, 2013, pages 1003-1010. IEEE Computer Society, 2013. ISBN 978-0-7695-4989-7. doi: 10.1109/CVPR.2013.134. URL http://dx.doi.org/10.1109/CVPR.2013.134.

[16] Christine Guillemot and Olivier Le Meur. Image inpainting : Overview and recent advances. IEEE Signal Process. Mag., 31(1):127-144, 2014. doi: 10.1109/MSP.2013. 2273004. URL http://dx.doi.org/10.1109/MSP . 2013.2273004. 
[17] Adrian Jarabo, Belen Masia, and Diego Gutierrez. Efficient propagation of light field edits. In Proc. of the V Ibero-American Symposium in Computer Graphics, SIACG 2011, pages 75-80, 2011. ISBN 978-972-98464-6-5.

[18] Marc Levoy and Pat Hanrahan. Light Field Rendering. In John Fujii, editor, Proceedings of the 23rd Annual Conference on Computer Graphics and Interactive Techniques, SIGGRAPH 1996, New Orleans, LA, USA, August 4-9, 1996, pages 31-42. ACM, 1996. ISBN 0-89791-746-4. doi: 10.1145/237170.237199. URL http: //doi.acm.org/10.1145/237170.237199.

[19] Ren Ng. Digital Light Field Photography. PhD thesis, Stanford, CA, USA, 2006. AAI3219345.

[20] Deepak Pathak, Philipp Krähenbühl, Jeff Donahue, Trevor Darrell, and Alexei Efros. Context encoders: Feature learning by inpainting. 2016.

[21] P. Perona and J. Malik. Scale-space and edge detection using anisotropic diffusion. IEEE Trans. Pattern Anal. Mach. Intell., 12(7):629-639, July 1990. ISSN 0162-8828. doi: 10.1109/34.56205. URL http://dx. doi .org/10.1109/34.56205.

[22] Leonid I. Rudin, Stanley Osher, and Emad Fatemi. Nonlinear total variation based noise removal algorithms. Phys. D, 60(1-4):259-268, November 1992. ISSN 01672789. doi: 10.1016/0167-2789(92)90242-F. URL http://dx.doi.org/10 . $1016 / 0167-2789(92) 90242-\mathrm{F}$.

[23] Steven M Seitz and Kiriakos N Kutulakos. Plenoptic Image Editing. International Journal of Computer Vision, 48(2):115-129, 2002. doi: 10.1023/A:1016046923611. URL http://dx.doi.org/10.1023/A:1016046923611.

[24] David Tschumperlé and Rachid Deriche. Vector-valued image regularization with pdes: A common framework for different applications. IEEE Trans. Pattern Anal. Mach. Intell., 27(4):506-517, 2005. doi: 10.1109/TPAMI.2005.87. URL http: //dx.doi.org/10.1109/TPAMI.2005.87.

[25] Sven Wanner and Bastian Goldluecke. Variational Light Field Analysis for Disparity Estimation and Super-Resolution. IEEE Trans. Pattern Anal. Mach. Intell., 36(3): 606-619, 2014. doi: 10.1109/TPAMI.2013.147. URL http: // dx. doi .org/10 . $1109 /$ TPAMI.2013.147.

[26] Joachim Weickert. Anisotropic diffusion in image processing. Teubner, 1998. ISBN 978-3-519-02606-8. URL http://d-nb.info/952577437.

[27] Philippe Weinzaepfel, Jerome Revaud, Zaid Harchaoui, and Cordelia Schmid. DeepFlow: Large displacement optical flow with deep matching. In IEEE Intenational Conference on Computer Vision (ICCV), Sydney, Australia, December 2013. URL http://hal.inria.fr/hal-00873592.

[28] Williem, Ki Won Shon, and In Kyu Park. Spatio-angular consistent editing framework for 4D light field images. Multimedia Tools and Applications, 75(23):16615-16631, 2016. ISSN 1573-7721. doi: 10.1007/s11042-016-3754-y. URL http: / / dx . doi . org/10.1007/s11042-016-3754-y. 
[29] F. L. Zhang, J. Wang, E. Shechtman, Z. Y. Zhou, J. X. Shi, and S. M. Hu. Plenopatch: Patch-based plenoptic image manipulation. IEEE Transactions on Visualization and Computer Graphics, PP(99):1-1, 2016. ISSN 1077-2626. doi: 10.1109/TVCG.2016. 2532329. 